\title{
Síntese, por reação de combustão em forno de microondas, de nanoferritas de níquel dopadas com cromo
}

\section{(Synthesis of nickel nanoferrites doped with chromium by microwave assisted combustion reaction)}

\author{
A. C. F. M. Costa ${ }^{1}$, P. Sarubo-Junior ${ }^{3}$, D.A. Vieira ${ }^{1}$, V.J.Silva ${ }^{1}$, T. S. Barros ${ }^{1}$, \\ D.R. Cornejo ${ }^{2}$, R.H.G.A.Kiminami ${ }^{3}$ \\ ${ }^{I}$ Departamento de Engenharia de Materiais, Universidade Federal de Campina Grande, \\ Av. Aprígio Veloso, 882, Bodocongó, Campina Grande, $P B$ \\ ${ }^{2}$ Instituto de Física, Universidade de S. Paulo, S. Paulo, SP, 05508-900 \\ ${ }^{3}$ Departamento de Engenharia de Materiais, Universidade Federal de S. Carlos, \\ Rod. Washington Luiz, km 235, S. Carlos, SP, 13656-905 \\ anacristina@dema.ufcg.edu.br, ruth@power.ufscar.br
}

\begin{abstract}
Resumo
Pós nanométricos de ferritas de níquel dopadas com cromo foram sintetizados por reação de combustão em forno de microondas. A influência da concentração de $\mathrm{Cr}^{3+}$ nas proporções de $\mathrm{x}=0$; 0,5 e 1 no sistema $\mathrm{NiCr}_{\mathrm{x}} \mathrm{Fe}_{2-\mathrm{x}} \mathrm{O}_{4}$ foi investigada. Os pós foram preparados de acordo com o conceito da química dos propelentes e aquecidos em forno microondas com potência $980 \mathrm{~W}$. Os pós sintetizados foram caracterizados por difratometria de raios X, adsorção de nitrogênio (método BET), microscopia eletrônica de varredura, picnometria de hélio e medidas magnéticas. Os resultados mostraram que foi possível obter nanopós de ferritas de níquel dopadas com cromo e que a elevação da concentração de cromo causou um aumento no tamanho de partículas ( $15 \mathrm{~nm}$ para $55 \mathrm{~nm}$ ) e redução nos parâmetros magnéticos.
\end{abstract}

Palavras-chave: síntese, reação de combustão, microondas, nanoferritas.

\begin{abstract}
Chromium-doped nickel nanoferrite powder was prepared by combustion reaction, using microwave energy as the external heating source, and the influence of the $\mathrm{Cr}^{3+}$ concentration on the $\mathrm{NiCr}_{x} \mathrm{Fe}_{2-x} \mathrm{O}_{4}$ system was investigated. The powder, which was prepared according to the concept of propellant chemistry, was heated in a microwave oven under a power of $980 \mathrm{~W}$. The resulting material was characterized by XRD, BET, SEM, helium picnometry and magnetic measurements. The results indicate that it was possible to obtain chromium-doped nickel ferrite nanopowders and that raising the chromium concentration caused an increase in particle size (from 15 $\mathrm{nm}$ to $55 \mathrm{~nm}$ ), and a reduction in the magnetic parameters.
\end{abstract}

Keywords: synthesis,combustion reaction, microwave, nanopowders

\section{INTRODUÇÃO}

As ferritas de níquel são materiais cerâmicos ferrimagnéticos e apresentam estrutura cristalina tipo espinélio inverso. São consideradas materiais magnéticos moles, pois apresentam baixo campo coercitivo e elevada permeabilidade [1]. Esse tipo de material é comumente produzido por meio da mistura de óxidos, que consiste na mistura de pós-precursores e uma posterior reação no estado sólido a elevadas temperaturas $\left(>1200{ }^{\circ} \mathrm{C}\right)$ [2]. Apesar do método de mistura de óxido cerâmico ser relativamente simples e o mais utilizado industrialmente, ele apresenta desvantagens inerentes, como: impurezas acrescidas durante o processo de moagem; baixo controle da composição; baixa homogeneidade química; tamanhos excessivamente grosseiros das partículas, comprometendo a microestrutura e propriedades finais desses materiais [3-8]. Para contornar os problemas advindos do método de mistura de óxidos, métodos químicos em escala de laboratório, tais como: sol-gel [9], co-precipitação [10], hidrotérmica [11], microemulsão [12], reação por combustão [13, 14], entre outros, foram desenvolvidos, visando obter principalmente o controle da homogeneidade química, morfológica e granulométrica dos pós, garantindo, assim, o controle da microestrutura e propriedades finais do material. $\mathrm{O}$ método de reação por combustão vem se destacando nos últimos anos, devido principalmente á facilidade de sua aplicação, ao custo relativamente baixo, ao controle da homogeneidade química, por requerer poucas etapas e curto tempo de duração [3-8]. 
O processo de síntese de reação por combustão de pós utilizando-se a energia de microondas é fundamentalmente diferente do processo convencional com relação às condições dos mecanismos de aquecimento. No forno de microondas, $o$ calor é gerado uniformemente em todo o volume da amostra pela interação instantânea das microondas com a solução. Em um aquecimento convencional, o calor é gerado por meio dos elementos de aquecimento e, então, transferido para a superfície da amostra [15].

A síntese por microondas tem diversas vantagens sobre os métodos convencionais de aquecimento, tais como: aquecimento rápido e uniforme, temperaturas mais baixas de síntese e redução de custos em termos de energia e tempo [16-18]. Assim, neste contexto este trabalho tem como objetivo a síntese por reação de combustão em microondas e caracterização de ferritas de níquel dopadas com cromo.

\section{MATERIAIS E MÉTODOS}

Asíntese dos pós do sistema $\mathrm{NiCr}_{x} \mathrm{Fe}_{(2-x)} \mathrm{O}_{4}$, ondex $=1,0,0,5$ e $0,0 \% \mathrm{~mol} \mathrm{Cr}^{3+}$ foi feita pelo método de reação de combustão em microondas com $\mathrm{Ni}\left(\mathrm{NO}_{3}\right)_{2} \cdot 6 \mathrm{H}_{2} \mathrm{O}, \mathrm{Cr}\left(\mathrm{NO}_{3}\right)_{3} \cdot 9 \mathrm{H}_{2} \mathrm{O}$, e $\mathrm{Fe}\left(\mathrm{NO}_{3}\right)_{3} \cdot 9 \mathrm{H}_{2} \mathrm{O}$ como reagentes precursores e fontes de cátions, e a uréia $\left[\mathrm{CO}\left(\mathrm{NH}_{2}\right)_{2}\right]$ como combustível, em um cadinho de sílica vítrea como recipiente. A mistura inicial (composição estequiométrica) foi calculada de acordo com as valências dos elementos reativos, de modo a favorecer a relação oxidante/combustível $=1$, de acordo com a química dos propelentes [19]. As reações de combustão ocorreram dentro de um forno de microondas Panasonic, potência 980 W. As amostras foram designadas M0, M0,5 e M1 para os sistemas com $\mathrm{x}=0,0,0,5$ e $1,0 \% \mathrm{~mol} \mathrm{Cr}^{3+}$, respectivamente. A temperatura máxima e o tempo da chama de combustão foram determinados em um espectrofotômetro Perkin Elmer Spectrum GX $\left( \pm 2{ }^{\circ} \mathrm{C}\right)$ e um cronômetro digital Condor, respectivamente. Os pós resultantes apresentaram-se na estrutura de flocos porosos, pouco volumosos e de coloração marrom escuro; posteriormente, foram desaglomerados em almofariz de ágata, peneirados em malha 200 mesh (74 $\mu \mathrm{m})$, e depois submetidos à caracterização. A análise de difração de raios $\mathrm{X}$ foi feita em um difratômetro de raios $\mathrm{X}$ Siemens D5000 com radiação Cuka. Os difratogramas foram obtidos na faixa $2 \theta$ de 10 a $60^{\circ}$ a $25^{\circ} \mathrm{C}$. A morfologia dos pós foi analisada por meio de microscopia eletrônica de varredura em um microscópio Zeiss DSM 940. Para a determinação da área superficial específica foi utilizado o método de adsorção de nitrogênio/hélio desenvolvido por Brunauuer, Emmett e Teller (BET) em um equipamento Gemini 2370 (Micromeritics). Os resultados da área superficial específica foram utilizados para calcular o tamanho de partícula utilizando a equação:

$$
\mathrm{D}_{\mathrm{BET}}=\frac{6}{\mathrm{D}_{\mathrm{t}} \mathrm{S}_{\mathrm{BET}}}
$$

na qual $\mathrm{D}_{\mathrm{Bet}}$ é o diâmetro esférico equivalente $(\mathrm{nm}), \mathrm{D}_{\mathrm{t}} \mathrm{a}$ densidade teórica da ferrita $\left(\mathrm{g} / \mathrm{cm}^{3}\right)$ e $\mathrm{S}_{\mathrm{Bet}}$ a área superficial $\left(\mathrm{m}^{2} / \mathrm{g}\right)$. A densidade real foi determinada num picnômetro a hélio Accupyc 1330 (Micromeritics) e as propriedades magnéticas (campo coercivo, magnetização de saturação, e magnetização remanente) em um magnetômetro de gradiente alternado (AGM), operando em campo aplicado até +6000 Oe à temperatura ambiente.

\section{RESULTADOS E DISCUSSÃO}

As análises das características dos nanopós sintetizados por reação de combustão em microondas foram feitas em função da concentração do íon $\mathrm{Cr}^{3+}$ no sistema $\mathrm{NiFe}_{2-\mathrm{x}} \mathrm{Cr}_{\mathrm{x}} \mathrm{O}_{4}$ com $\mathrm{x}=1,0,0,5$ e 0 mol. As Figs. 1a e $1 \mathrm{~b}$ mostram os valores e a curva de tendência para temperatura máxima e do tempo de chama de ignição em função do aumento da concentração de $\mathrm{Cr}^{3+}$ no sistema $\mathrm{NiCr}_{x} \mathrm{Fe}_{2-\mathrm{x}} \mathrm{O}_{4}$ para os nanopós preparados por reação de combustão usando forno microondas como fonte de aquecimento.

A elevação da concentração de $\mathrm{Cr}^{3+}$ causou um aumento na temperatura máxima de ignição e redução do tempo de chama de combustão. As reações de síntese por combustão foram favorecidas pela síntese ter sido assistida em forno de microondas e o efeito da adição do $\mathrm{Cr}$ no sistema analisado ter sido nitidamente observado. $\mathrm{O}$ fato de o cromo tender a elevar a temperatura da síntese deve ser provavelmente causado pela razão de o calor de formação do cromo $(-1139,7 \mathrm{~kJ} / \mathrm{mol})$ ser inferior ao de formação do ferro $(-824,2 \mathrm{~kJ} / \mathrm{mol})$, o que necessita gerar maior temperatura para ocorrer completa formação da fase. Esse comportamento já foi reportado no estudo do sistema ferrita Ni-Zn dopado com cromo por reação de combustão convencional, usando um becker de pirex e aquecimento direto em uma placa quente a $480{ }^{\circ} \mathrm{C}$ para a síntese.

A Fig. 2 apresenta os difratogramas de raios $\mathrm{X}$ dos pós obtidos por reação de combustão em forno de microondas. Os nanopós apresentaram apenas a formação da fase cristalina cúbica do espinélio inverso $\mathrm{NiFe}_{2} \mathrm{O}_{4}$ (JCPDF 05-0669). O nanopó do sistema M0 resultou em menor cristalinidade quando comparado com as composições dopadas com 0,5 e $1,0 \mathrm{~mol} \mathrm{de} \mathrm{Cr}^{3+}$. Isso se deu em função da menor temperatura de combustão $\left(560{ }^{\circ} \mathrm{C}\right)$ alcançada nesta composição. Esses resultados confirmam a forte influência do cromo e do tipo de aquecimento, indicando que o aumento da concentração de $\mathrm{Cr}^{3+}$ e a própria uniformidade de aquecimento no forno de microondas favoreceram o aumento da temperatura e redução do tempo de combustão, levando à formação de nanopós com alta cristalinidade e completa formação da fase ferrita, sem a presença de traços da fase hematita.

A Tabela I apresenta os resultados das características dos nanopós sintetizados por reação de combustão em forno microondas. Observamos que o aumento da concentração de $\mathrm{Cr}^{3+}$ no sistema da ferrita de níquel levou a uma acentuada redução na área superficial e, consequentemente, a um aumento no tamanho de partícula. 

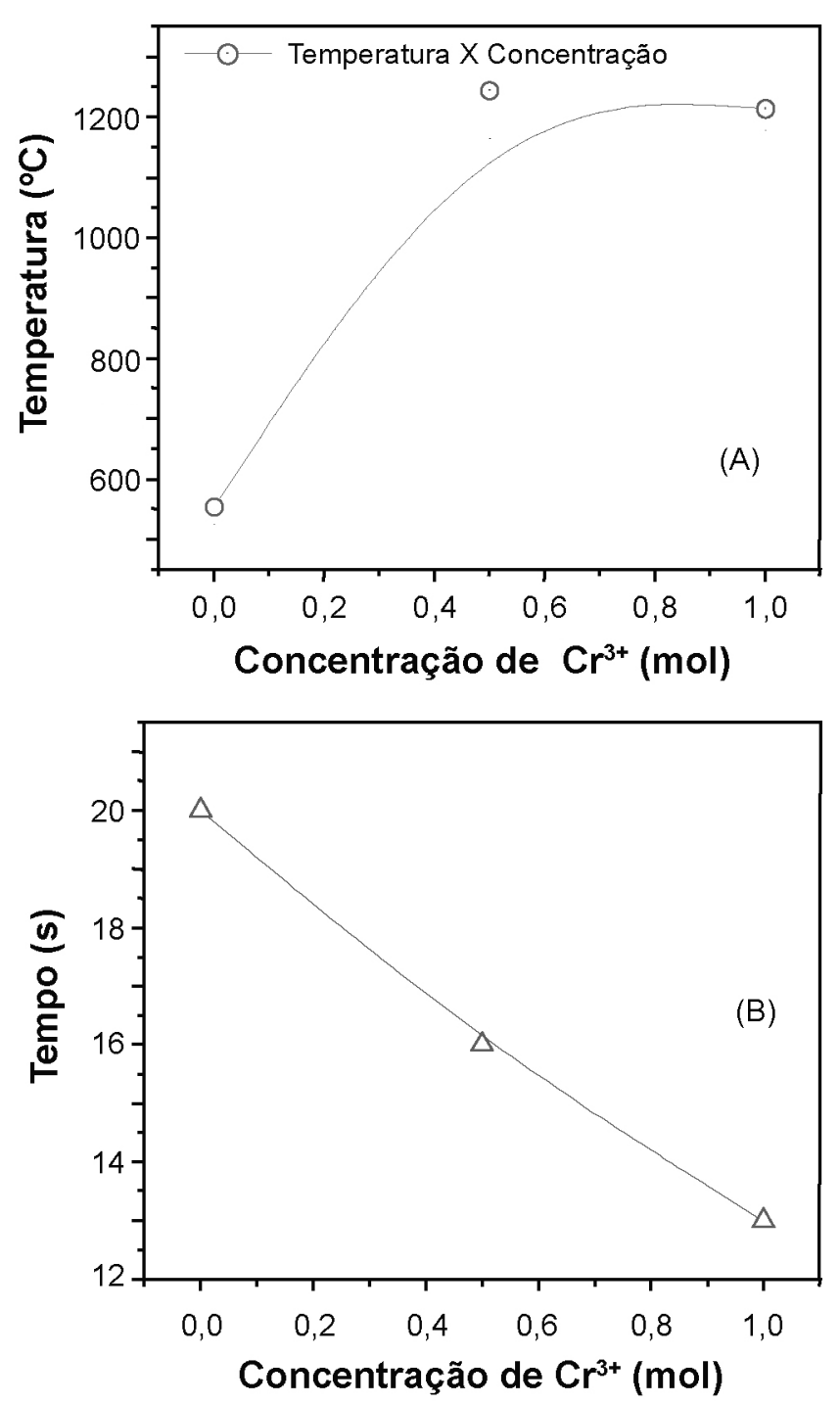

Figura 1: Temperatura da chama de combustão (a) e tempo de chama (b) das ferritas de níquel dopadas com cromo com composição de $\mathrm{NiCr}_{x} \mathrm{Fe}_{(2-x)} \mathrm{O}_{4}, \mathrm{x}=1,0 ; 0,5$ e $0 \% \mathrm{~mol} \mathrm{de} \mathrm{Cr}^{3+}$, obtidas por reação de combustão em forno de microondas.

[Figure 1: Combustion flame temperatures (a) and time (b) of the Ni-doped ferrites with nominal compositions $\mathrm{NiCr}_{x} \mathrm{Fe}_{(2-x)} \mathrm{O}_{4}$, $x=1.0,0.5$ and $0 \mathrm{~mol} \% \mathrm{Cr}^{3+}$, obtained by microwave assisted combustion reaction.]

Estes resultados demonstram o efeito inerente da adição do $\mathrm{Cr}$ nas características estruturais das ferritas de níquel, a conseqüente alteração nos parâmetros de temperatura e o tempo de reação.

As Figs 3a, b e c apresentam as micrografias para os nanopós de ferrita preparados por reação de combustão em forno microondas. As nanopartículas dos três sistemas são inferiores a $200 \mathrm{~nm}$, que favoreceu a formação de aglomerados moles de nanopartículas.

Em todos os sistemas estudados observamos que as nanopartículas apresentaram morfologia e tamanho uniformes, constituídas apenas por nanopartículas primárias aglomeradas. É sabido que quanto menor o tamanho de partículas, maior a sua tensão superficial, o que gera força

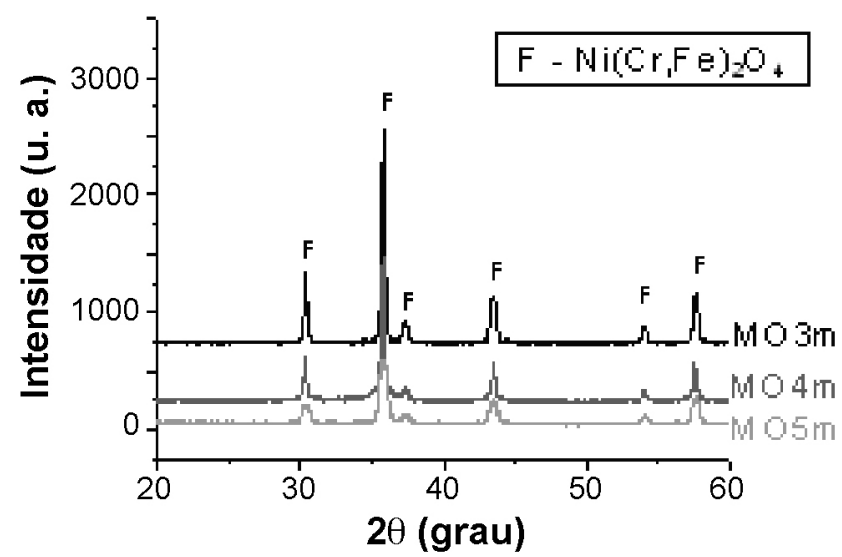

Figura 2: Difratogramas de raios $\mathrm{X}$ dos nanopós de ferrita de níquel dopados com cromo obtidos por reação de combustão em forno microondas.

[Figure 2: X-ray diffraction patterns of chromium-doped nickel nanoferrite powders obtained by microwave assisted combustion reaction.]

motriz para favorecer o aumento do estado de aglomeração e/ou agregação. Experimentalmente (no processo de peneiramento), observamos que quanto maior a concentração de $\mathrm{Cr}^{3+}$ no sistema, menos friável, ou seja, mais difícil foi a desaglomeração do pó. Por meio dessas micrografias, podemos, então, observar que, para as composições M0 e M0,5, houve a formação de aglomerados moles (fácil desaglomeração) com morfologia irregular e um aspecto poroso constituído de partículas bastante finas; e que o estado de aglomeração tornou-se mais duro à medida que se elevou a concentração de íons $\mathrm{Cr}^{3+}$ na ferrita de níquel $\left(\mathrm{NiFe}_{2} \mathrm{O}_{4}\right)$, amostra M1.

As Figs. 4a, b e c apresentam as curvas de histerese para os sistemas preparados por reação de combustão em forno microondas. Observamos que a magnetização de saturação (Ms) para os pós M0, M0,5 e M1 foram 37, 12 e 3 emu/g, respectivamente. Por meio das curvas de histerese $\sigma x H$, podemos observar que o aumento da concentração de $\mathrm{Cr}^{3+}$ causou um alargamento do laço de histerese e redução da magnetização de saturação, ou seja, compromete o comportamento de materiais magnéticos moles (baixo campo coercitivo, baixa perda por histerese e valores de

Tabela I - Tamanho de partícula estimado a partir da área superficial específica.

[Table I - Particle size after surface area measurements.]

\begin{tabular}{cccc}
\hline Sistemas & $\mathrm{M} 0$ & $\mathrm{M} 0,5$ & $\mathrm{M} 1$ \\
\hline & $\mathrm{NiFe}_{2} \mathrm{O}_{4}$ & $\mathrm{NiCr}_{0,5} \mathrm{Fe}_{1,5} \mathrm{O}_{4}$ & $\mathrm{NiCr}_{1,0} \mathrm{Fe}_{1,0} \mathrm{O}_{4}$ \\
$\begin{array}{c}\text { Área superficial } \\
(\mathrm{BET})\left[\mathrm{m}^{2} / \mathrm{g}\right]\end{array}$ & 72,8 & 68,7 & 20,0 \\
$\begin{array}{c}\text { Tamanho de } \\
\text { partícula* }[\mathrm{nm}]\end{array}$ & 15 & 16 & 55 \\
\hline * valor calculado & & &
\end{tabular}



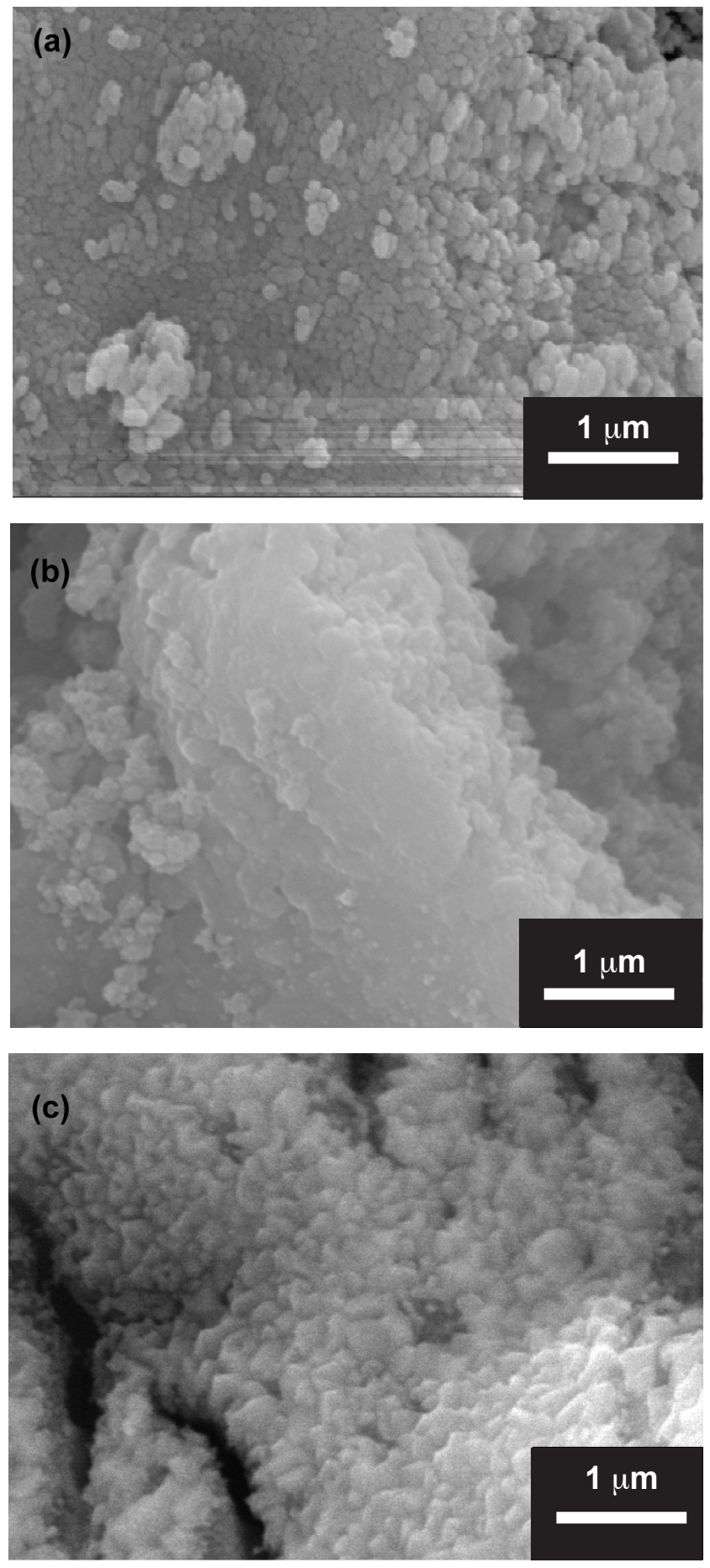

Figura 3: Micrografias obtidas em microscópio eletrônico de varredura dos nanopós de ferritas dopadas com cromo: (a) M0 $\left(\mathrm{NiFe}_{2} \mathrm{O}_{4}\right)$, (b) M0,5 ( $\left.\mathrm{NiCr}_{0,5} \mathrm{Fe}_{1,5} \mathrm{O}_{4}\right)$ e (c) $\mathrm{M} 1 \mathrm{NiCr}_{1,0} \mathrm{Fe}_{1,0} \mathrm{O}_{4}$.

[Figure 3: SEM micrographs of chromium-doped nickel nanoferrite powders: (a) $\mathrm{MO}$ ( $\mathrm{NiFe}_{2} \mathrm{O}_{4}$ ), (b) $\mathrm{MO} .5$ ( $\mathrm{NiCr}_{0.5} \mathrm{Fe}_{1.5} \mathrm{O}_{4}$ ) e (c) $\mathrm{Ml}$ $\mathrm{NiCr}_{1.0} \mathrm{Fe}_{1.0} \mathrm{O}_{4} \cdot \mathrm{J}$

magnetização elevados).

A Tabela II mostra os dados magnéticos (campo coercivo, magnetização de saturação, magnetização remanente e perda por histerese) obtidos a partir das curvas $\sigma x H$.

O aumento da concentração de cromo reduziu
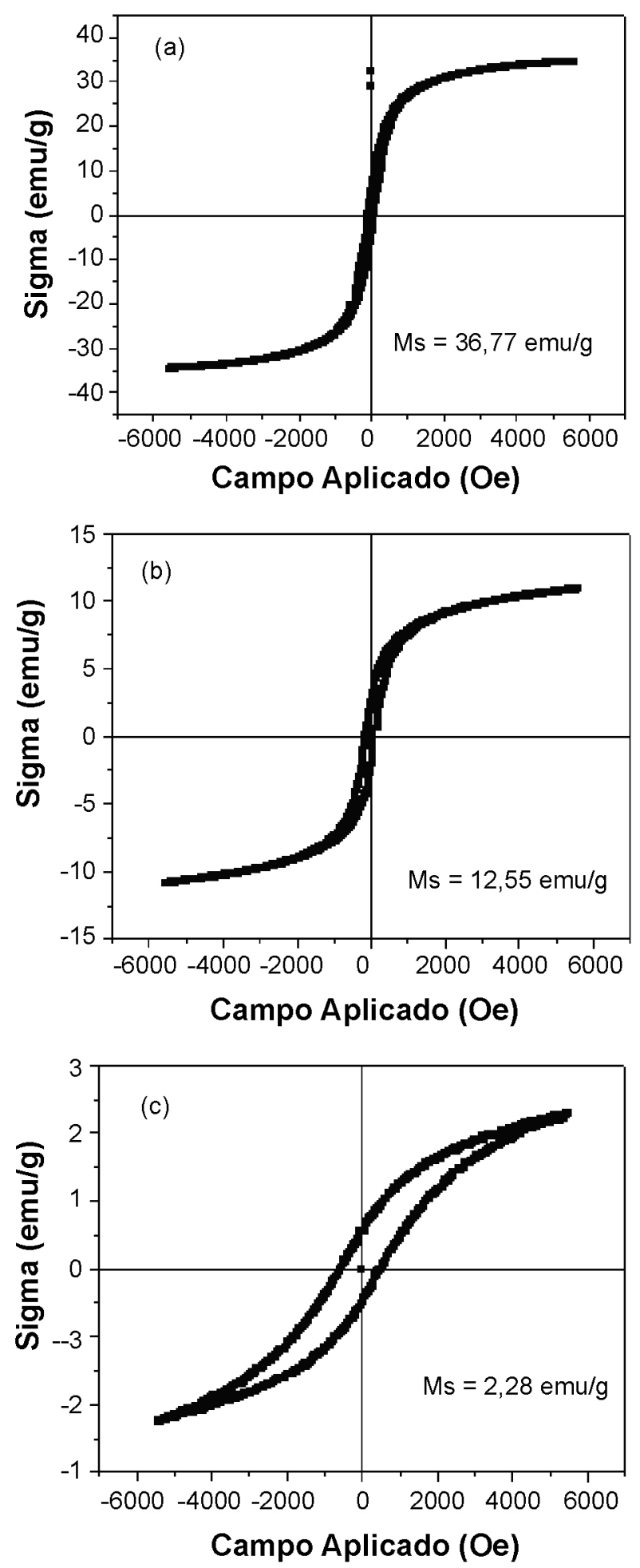

Figura 4: Curva de histerese à temperatura ambiente dos nanopós de ferritas de níquel dopadas com cromo: (a) $\mathrm{M} 0\left(\mathrm{NiFe}_{2} \mathrm{O}_{4}\right)$, (b) $\mathrm{M} 0,5\left(\mathrm{NiCr}_{0,5} \mathrm{Fe}_{1,5} \mathrm{O}_{4}\right)$ e (c) $\mathrm{M} 1\left(\mathrm{NiCr}_{1,0} \mathrm{Fe}_{1,0} \mathrm{O}_{4}\right)$.

[Figure 4: Room temperature hysteresis loops of chromiumdoped nickel nanoferrite powders: (a) $\mathrm{MO}\left(\mathrm{NiFe}_{2} \mathrm{O}_{4}\right)$, (b) M0.5 $\left(\mathrm{NiCr}_{0.5} \mathrm{Fe}_{1.5} \mathrm{O}_{4}\right)$ e (c) $\mathrm{MI} \mathrm{NiCr} \mathrm{Fe}_{1.0} \mathrm{Fe}_{1.0} \mathrm{O}_{4} . \mathrm{J}$

drasticamente a magnetização de saturação e aumentou excessivamente o campo coercivo. Comparando os sistemas com $x=1,0$ e 0,0 mol de cromo, verificamos uma redução 
Tabela II - Dados magnéticos dos nanopós sintetizados por reação de combustão em forno microondas.

[Table II - Magnetic parameters of powders obtained by microwave assisted combustion reaction.]

\begin{tabular}{cccc}
\hline & $\mathrm{M} 0$ & $\mathrm{M} 0,5$ & $\mathrm{M} 1$ \\
\hline & $\mathrm{NiFe}_{2} \mathrm{O}_{4}$ & $\mathrm{NiCr}_{0,5} \mathrm{Fe}_{1,5} \mathrm{O}_{4}$ & $\mathrm{NiCr}_{1,0} \mathrm{Fe}_{1,0} \mathrm{O}_{4}$ \\
$\mathrm{Hc}(\mathrm{Oe})$ & 92,4 & 141,6 & 563,8 \\
$\mathrm{Ms}(\mathrm{emu} / \mathrm{g})$ & 37 & 12 & 3 \\
$\mathrm{Mr}(\mathrm{emu} / \mathrm{g})$ & 4,4 & 2,2 & 0,6 \\
$\mathrm{Mr} / \mathrm{Ms}$ & 0,12 & 0,17 & 0,24 \\
\hline
\end{tabular}

na magnetização de aproximadamente 10 vezes. Isso indica que a substituição de 1,0 mol de cromo por ferro foi excessivamente alta, perdendo, assim, a característica de uma ferrita e se comportando mais como uma cromita, a qual não tem característica de materiais ferrimagnéticos e, sim, paramagnéticos. Deve-se salientar que a concentração de ferro para constituir um sistema ferrita deve ser igual ou superior a $70 \%$.

É sabido que a ferrita de níquel é um espinélio inverso, cuja célula unitária é representada pela formula $\left(\mathrm{Fe}_{1-\mathrm{x}}\right)\left[\mathrm{NiFe}_{1+\times}\right] \mathrm{O}_{4}$, onde os íons de $\mathrm{Fe}^{3+}$ estão igualmente distribuídos nas posições tetraédrica e octaédrica da rede. $\mathrm{O}$ íon $\mathrm{Cr}^{3+}$ é conhecido por ser um elemento paramagnético [21] cujo momento magnético de Bohr é $3 \mu \mathrm{B}$. O Fe ${ }^{3+} \mathrm{e}$ o $\mathrm{Ni}^{2+}$ são magnéticos, com momento magnético de Bohr $5 \mu \mathrm{B}$ e $2 \mu \mathrm{B}$, respectivamente. Considerando a forte preferência do íon $\mathrm{Cr}^{3+}$ pela posição octaédrica da rede espinélio [22] e supondo que o cromo substitui totalmente os íons $\mathrm{Fe}^{3+}$ nesta posição, teremos que a magnetização teórica (característica intrínseca) calculada da rede dos espinélios $\mathrm{NiFe}_{2} \mathrm{O}_{4}, \mathrm{NiFe}_{1,5} \mathrm{Cr}_{0,5} \mathrm{O}_{4}$ e $\mathrm{NiFe}_{1,0} \mathrm{Cr}_{1,0} \mathrm{O}_{4}$ é $16 \mu_{\mathrm{B}}$. $8 \mu_{\mathrm{B}} \mathrm{e} 0 \mu_{\mathrm{B}}$ respectivamente. Portanto, uma redução drástica nas propriedades magnéticas quando da substituição simultânea do cromo por ferro na rede espinélio da ferrita de níquel é esperado. Por outro lado, é sabido que as características extrínsecas como tamanho de grão e/ou partícula influenciam a área de domínio magnético e podem contribuir para o aumento da magnetização, pois quanto maior o tamanho da partícula e/ou dos grãos, menor o número de barreiras, favorecendo uma maior magnetização. Neste trabalho, verificamos que o aumento da concentração de cromo levou a um crescimento do tamanho da partícula, porém, com relação à magnetização, observamos uma queda acentuada e um aumento no campo coercivo, o que indicou que a característica intrínseca prevaleceu. Assim, ficou evidente a influência significativa do cromo em substituição aos íons de ferro na rede da ferrita de níquel tanto na síntese, quanto nas características morfológicas e magnéticas dos nanopós sintetizados em microondas.

\section{CONCLUSÃO}

A síntese por reação de combustão assistida em microondas foi favorável para a obtenção de pós nanocristalinos de ferritas de Ni dopadas com cromo. Todas as composições estudadas apresentaram apenas a formação da fase cristalina cúbica do espinélio inverso $\mathrm{NiFe}_{2} \mathrm{O}_{4}$. O aumento da concentração de cromo proporcionou aumento da temperatura e redução do tempo de chama de ignição, o que interferiu diretamente nas características estruturais das nanopartículas, causando redução da magnetização de saturação e aumento do campo coercivo.

Os sistemas de ferritas estudados $\left(\mathrm{NiCr}_{x} \mathrm{Fe}_{(2-x)} \mathrm{O}_{4}\right.$ $\mathrm{x}=1,0,0,5$ e $0 \% \mathrm{~mol} \mathrm{de} \mathrm{Cr}^{3+}$ ) apresentaram características magnéticas diferenciadas, de acordo com a quantidade de dopante utilizado, permitindo o uso desses materiais como magnéticos moles (permeável) e intermediários (meio de gravação magnética), com ciclos de histerese relativamente estreitos.

\section{AGRADECIMENTOS}

À FAPESP pelo apoio financeiro (Proc. 05/60095-9) e ao CNPq pelas bolsas concedidas.

\section{REFERÊNCIAS}

[1] A. Verma, T. C. Goel, R. G. Mendiratta, P. Kishan, J. Magn. Magn. Mater. 208 (2000) 13-19.

[2] K. Kondo, T. Chiba, S. Yamada, J. Magn. Magn. Mater. 254-255 (2003) 541-543.

[3] A. C. F. M. Costa, E. Tortella, M. R. Morelli, R. H. G. A. Kiminami, J. Metastable and Nanocryst. Mater. 14 (2002) 57-63.

[4] Y. Zhang, G. C. Stangle, J. Mater. Res. 9,8 (1994) 1997 2004.

[5] A. C. F. M. Costa, Tese de Doutorado, Departamento de Engenharia de Materiais, Universidade Federal de S. Carlos, S. Carlos (2002).

[6] A. C. F. M. Costa, R. H. G. A. Kiminami, M. R. Morelli, J. Mter. Sci. 42 (2007) 779-783.

[7] M.A. F. Ramalho, A. C. F. M. Costa, L Gama, R. H. G.A. Kiminami, E. P. Hernandez, D. R. Cornejo, S. M. Rezende, Mater. Sci. Forum 530-531 (2006) 637-642.

[8] A. C. F. M. Costa, M. R. Morelli, R. H. G. A. Kiminami, Combustion Synthesis Processing of Nanoceramics, Handbook of Nanoceramics and their Based Nanodevices Ed. Am. Sci. Publ. (2008).

[9] C. Otero Areán, M. Penarroya Mentruit, A. J. López López, J. B. Parra, Physicochem. Eng. Aspects 180 (2001) 253-258.

[10] P. Cesteros, F. Salagre, J. E. Medina, Sueiras, Chem. Mater. 12 (2000) 331.

[11] F. Meyer, R. Hemplemann, S. Mathur, M. Veith, J. Mater. Chem. 9 (1999) 1955.

[12] S. Ono, S Hironi, J. Am. Ceram. Soc. 80, 10 (1997) 2533.

[13] T. Minami, J. Alloys Compds. 315 (2001) 123-128.

[14] Y. Zhang, G. C. Stangle, J. Mater. Res. 9, 8 (1994) 1997-2004.

[15] Yen-Pei Fu, Yu-Hsiu Su, She-Huang Wu , Cheng- 
Hsiung Lin, J.Alloys Compds. 426 (2006) 228-234.

[16] N. S. L. S. Vasconcelos, V. Bouquet, S. M. Zanetti, E. R. Leite, E. Longo, M. I. Bernardi, A. Perrin, M. GuillouxViry, J. A. Varela, Cerâmica 50 (2004) 128-133.

[17] R. H. G. A. Kiminami, M. R. Morelli, D. C. Folz, D. E. Clark, Am. Ceram. Soc. Bull. 70, 3 (2000) 63-67.

[18] R. H. G. A. Kiminami, KONA Powder and Particle, 19 (2001) 156-165.

[19] S. R. Jain, K. C. Adiga, V. Pai Verneker, Combustion and Flame 40 (1981) 71-79.

[20] A. C. F. M Costa, L. Gama, R. H. G. A. Kiminami, M.
A. F Ramalho, C. O. Paiva-Santos, V. H. S Utuni, Effect of substituting chromium in nickel ferrite: synthesis and characterization, Proc. Fourth Int. Latin-American Conf. Powder Technology, S. Paulo, SP (2003) cd-rom, p. 12971298.

[21] S. M. Rezende, A física de materiais e dispositivos eletrônicos, Ed. Universidade Federal de Pernambuco, Recife, PE (1991) 922-924.

[22] R. C. O'Handley, Modern Magnetics Materials: Principles and Applications. New York: John Wiley \& Sons, Inc. (1999).

(Rec. 27/03/2008, Ac. 20/06/2008) 